\title{
Frequency Doubling a Compact Stable External Cavity Diode Laser Using a Novel PPLN Waveguide
}

\author{
Stephen G. Lynch, Lewis G. Carpenter, Sam A. Berry, Teresa I. Ferreiro, Christopher Holmes, \\ Corin B. E. Gawith, Peter G. R. Smith \\ Optoelectronics Research Centre, University of Southampton, University Road, Southampton, SO17 1BJ, UK
}

There is increasing demand for low noise, narrow linewidth lasers for applications including spectroscopy, ion traps for optical clocks and gravitational waves measurements. External cavity diode lasers (ECDL) can provide a compact, low cost solution; however these are typically available at a limited range of optical frequencies. The increase in applications requiring robust laser systems (e.g. space borne gravitational wave measurements) has led to a need for alternatives at frequencies where free space lasers have previously been utilised.

We have previously demonstrated a novel planar platform using flamed hydrolysis deposition (FHD) to bond optical fibers to silicon wafers and form integrated optical fiber (IOF) into which Bragg gratings can be written at precisely selected wavelengths using a direct UV writing technique [1]. Low noise, narrow linewidth external cavity diode lasers have been constructed in a bench-top system built on translation stages using these IOF gratings as the external cavity for gain chips at wavelengths corresponding to selected gas absorption lines, e.g. acetylene [2]. Here we present results for an IOF-based ECDL that has been constructed into a compact and portable $1560 \mathrm{~nm}$ laser, see Fig. 1(a) and (b), with reduced susceptibility to low frequency RIN to offer higher stability. Frequency doubling is achieved by a periodically poled magnesium doped lithium niobate (PPLN) waveguide, see Fig. 1(c). The PPLN waveguides are fabricated via a bonding, thinning and ultra precision dicing route, this creates a $\mathrm{LiNbO}_{3}$-on-Si structure. Second harmonic generation is achieved with a $19.6 \mu \mathrm{m}$ period grating. This laser has been frequency doubled to $780 \mathrm{~nm}$, see Fig. 1(d), so it can be temperature tuned to a rubidium absorption line for locking. The FHD and UV writing techniques used to build this laser enable significant flexibility for the wavelengths achievable from these stable lasers which, with the use of PPLN for frequency up/down conversion, can offer stable sources for applications at optical and IR frequencies not previously accessible with compact, low-cost laser systems.

(a)

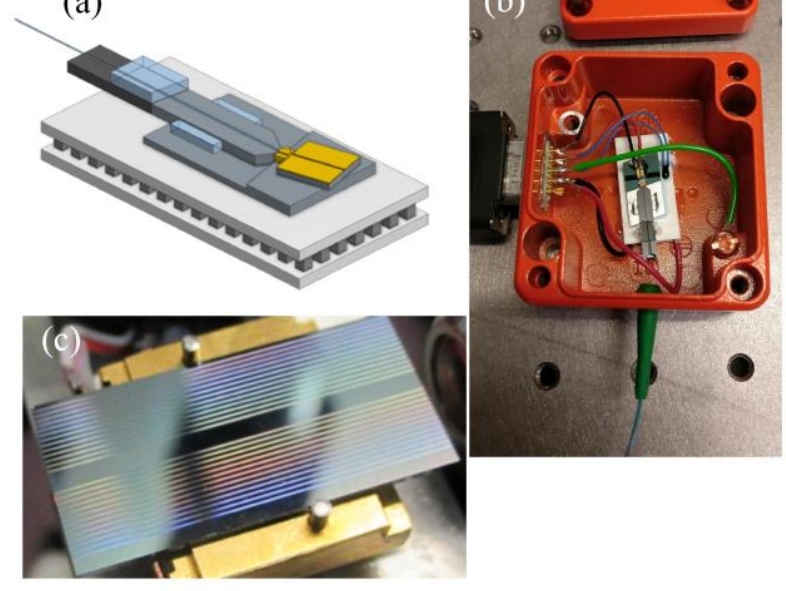

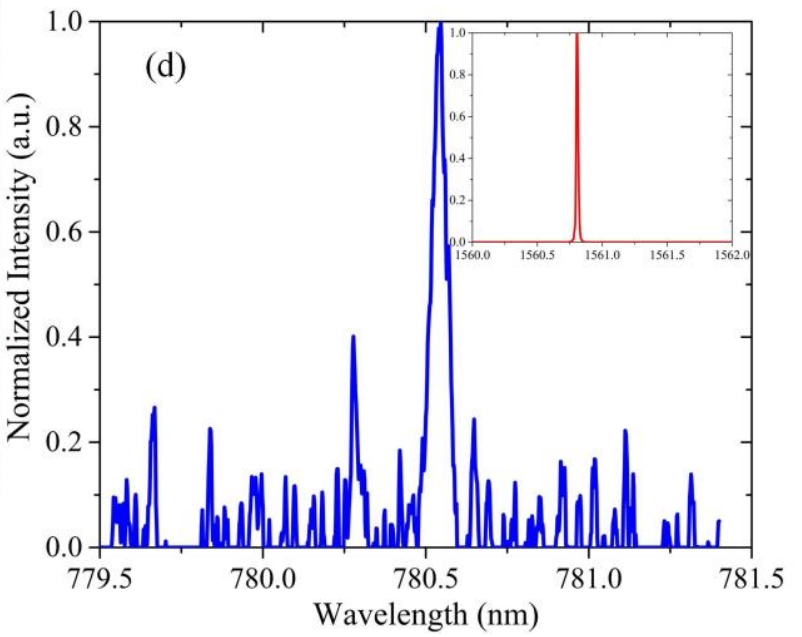

Fig. 1. (a) CAD image of the ECDL with fiber output. (b) Photograph of packaged laser. (c) Photograph PPLN waveguide array. (d) Spectra for the frequency doubled output from the PPLN and the ECDL (inset).

The low frequency intensity noise (sub-100 Hz) was significantly reduced when compared with previous lasers [2], and we will present further results demonstrating the low noise and high stability that can be achieved for the output of a frequency doubled IOF-based ECDL. We will also show locking of the ECDLs for increasing long-term stability.

\section{References}

[1] C. Holmes, J. C. Gates, and P. G. R. Smith, "Planarised optical fiber composite using flame hydrolysis deposition demonstrating an integrated FBG anemometer.” Opt. Express 22, 32150 (2014).

[2] S. G. Lynch, C. Holmes, S. A. Berry, J. C. Gates, A. Jantzen, T. I. Ferreiro, and P. G. R. Smith, "Narrow linewidth external cavity diode laser using UV-written gratings in an integrated optical fiber platform.” Opt. Express 24, 8391 (2016). 